\title{
Fabrication of Submicrometer InGaAs/AlAs Resonant Tunneling Diode Using a Tri-layer Soft Reflow Technique with Excellent Scalability
}

\author{
M. A. Md Zawawi, KaWa Ian, J. Sexton and M. Missous, Senior Member, IEEE
}

\begin{abstract}
A tri-layer soft reflow fabrication method using solvent vapour that resulted in a sub-micrometer resonant tunneling diode is reported in details. The processing steps are simple, time efficient and are all based on conventional i-line photolithography. The tri-layer soft reflow technique is able to shrink the emitter lateral width from $1 \mu \mathrm{m}$ down to $0.35 \mu \mathrm{m}(65 \%$ reduction) using a solvent at a very low temperature $\left(<50{ }^{\circ} \mathrm{C}\right)$. Studies of device's peak current density $\left(J_{P}\right)$ suggests that excellent scalability is achieved as the emitter area reduces from $\sim 29 \mu \mathrm{m}^{2}$ down to $\sim 0.5 \mu \mathrm{m}^{2}$ with no significant increase in peak voltage $\left(V_{P}\right)$ due to high series resistance normally associated with submicrometer dimensions. The valley current $\left(I_{V}\right)$ however increases due to side-wall damage introduced by the reactive ion etching (RIE) process. As a result, the peak-tovalley-current ratio (PVCR) decreases from 5.0 (6.3) to 3.8 (4.1) in forward (reverse) direction as the emitter area decreases. We therefore successfully demonstrated the fabrication of a sub-micrometer RTD by using a tri-layer soft reflow technique that has the benefit of excellent scalability, high throughput, repeatable and a reliable low cost process.
\end{abstract}

Index Terms-InGaAs-AlAs, resonant tunneling diode, soft reflow, submicrometer processing.

\section{INTRODUCTION}

MONGST all high frequency electron devices, the A resonant tunneling diode (RTD) receives a lot of attention due to its simple structure and ultra high

M.A. Md Zawawi, Ka Wa Ian, J. Sexton and M. Missous are with the School of Electrical and Electronic Engineering, University of Manchester, Manchester, M13 9PL UK mohamad.mdzawawi@postgrad.manchester.ac.uk; m.missous@manchester.ac.uk). frequency capability. The growing interest in RTD, due to its ability to generate continuous wave $(\mathrm{CW})$ at terahertz frequencies, has seen many breakthroughs in this fields with excellent results [1-3]. To date, the highest room temperature fundamental oscillation of up to $1.31 \mathrm{THz}$ is achieved in thin-well $(3.9 \mathrm{~nm})$ resonant tunneling diodes based on strained InGaAs-AlAs [3]. Currently, the terahertz and sub-terahertz frequency proves its usefulness in various applications such as radio astronomy, medical imaging, surveillance and security, atmospheric and environmental monitoring as well as holding great promises for gigabit per second (Gb/s) wireless communication [4].

As a rule of thumb, any semiconductor device which operates at very high frequency needs to have very small lateral dimension (to minimise capacitance) and the RTD is no exception [5]. Therefore the need to process down to sub-micrometer RTD mesa area is a must.

Several works have been done related to submicrometer RTD, in order to realise a one-dimensional (1-D) devices. These one-dimensional RTDs have typical diameter of $100 \mathrm{~nm}$. The first attempt was pioneered by Reed et.al [6] from Texas Instrument back in late 80 's. In Reed's work, the RTD had a diameter of $100 \mathrm{~nm}$ to $200 \mathrm{~nm}$ patterned by electron-beam lithography (EB) and reactive ion etching (RIE) for sidewall formation. The material used was GaAs/AlGaAs/InGaAs (contacts/barriers/well). However, the resulting DC characteristics were rather disappointing with peak-to-valley-current ratio of 1.1 [7].

Further work on sub-micrometer RTDs was then carried out using focussed ion beams in order to achieve $\sim 100 \mathrm{~nm}$ diameter as demonstrated by Tarucha [8]. There are also sub-micrometer RTD fabricated by

0018-9383 (c) 2014 IEEE. Personal use of this material is permitted. Permission from IEEE must be obtained for all other users, including reprinting/ republishing this material for advertising or promotional purposes, creating new collective works for resale or redistribution to servers or lists, or reuse of any copyrighted components of this work in other works. DOI: 
photolithography with selective wet etching, however the metal contact was not put directly on top of the device in these studies [9]. The most recent and complex method of creating sub-micrometer RTD is by using nanowire or nanowhiskers using chemical beam epitaxy [7].

All the techniques mentioned above are rather complicated and expensive, and fairly time consuming with slow through-put especially when using electronbeam (EB) lithography. The method proposed in this work is simple, reproducible and provides an economical solution in order to obtain sub-micrometer feature sizes. This new process was first introduced for application in pHEMTs [10]. The technique, termed low temperature soft reflow, uses conventional i-line photolithography to define the lateral structure and solvent vapour to achieve sub-micrometer features.

However, in this work, the original technique with bilayer lift-off has been modified and extended to suit the processing of an RTD by employing a tri-layer lift-off technique. Since the RTD does not require a T-gate shape as in the case of a pHEMT, therefore to create a sub-micrometer RTD, the soft-reflow technique is combined with a trilayer lift-off technique that results in a straight top metal contact.

\section{SOFT REFLOW PRINCIPLE}

The main principle involves the absorption of vapour by the photoresist to expand its volume. The soft reflow process is done in a purpose -built closed chamber with a sample being placed at some distance above the solvent. The entire process is controllable through these parameters: solvent temperature, duration of reflow and thickness of the photoresist. While the solvent temperature and photoresist thickness are kept constant, the shrinking of the initial opening (L) is inversely related to reflow duration (time in seconds). In another word, as the reflow duration increases, more vapour will be absorbed by the photoresist on both sides of the opening, hence a smaller opening (L') is obtained. The amount of shrinkage is given by parameter $\Delta \mathrm{L}$. This soft reflow process is quick to perform and most beneficial especially in the absence of electron beam (EB) lithography to define the sub-micrometer lateral dimensions. Fig. 1 illustrates the principle of the soft reflow technique.

The photoresist that is readily coated onto the sample absorbs the vapour by a method of condensation. An isotropic expansion in terms of photoresist volume occurs on both sides of the opening and results in a reduced final dimension.

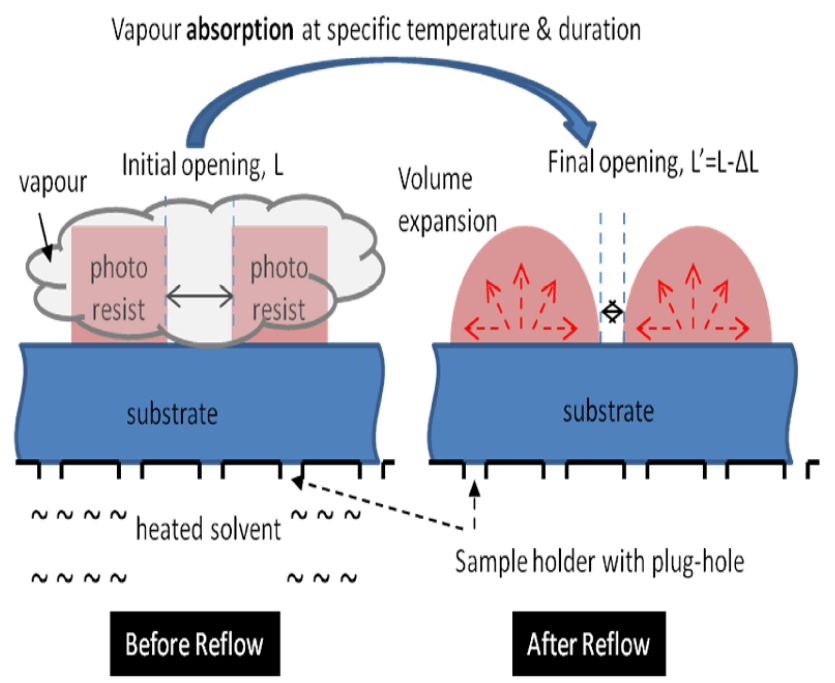

Fig. 1. Illustration of the soft reflow principle.

This work is an extension to the previously reported bi-layer soft reflow technique successfully performed on pHEMTs by K. W. Ian et al [11]. The trend of the feature size reduction against reflow time is almost similar for both bi-layer and tri-layer techniques as shown in Fig. 2. Slight differences between the two curves could be attributed to differences in ambient conditions, namely the temperature and humidity. Any changes in either one or both of these external parameters would cause changes in the solvent evaporation rate and vapour absorption rate.

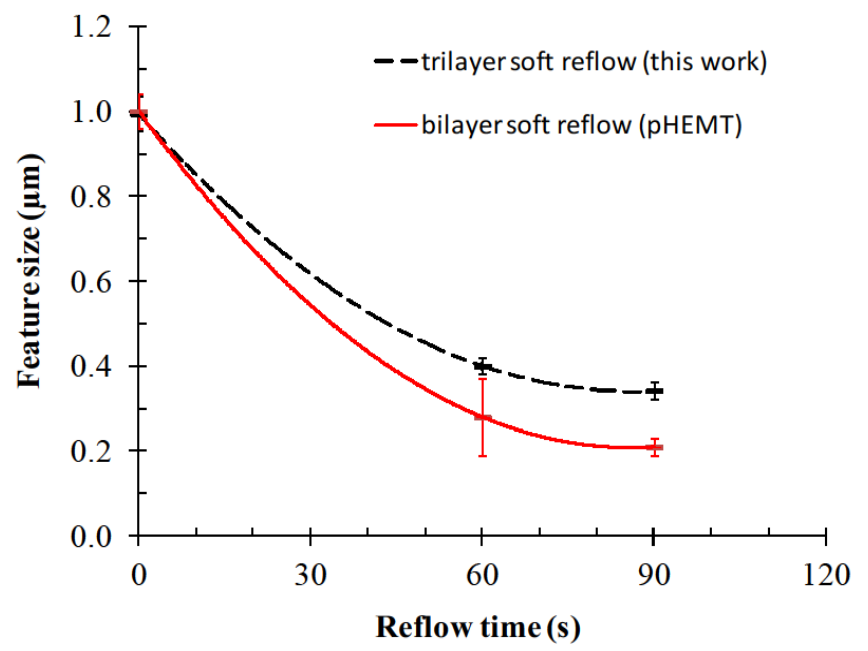

Fig. 2. Relationship between feature size and reflow time in this study in comparison to bi-layer soft reflow process for pHEMT [11].

0018-9383 (c) 2014 IEEE. Personal use of this material is permitted. Permission from IEEE must be obtained for all other users, including reprinting/ republishing this material for advertising or promotional purposes, creating new collective works for resale or redistribution to servers or lists, or reuse of any copyrighted components of this work in other works. DOI:

http://dx.doi.org/10.1109/TED.2014.2322107 


\section{SAMPLE PREPARATION AND PROCESSING}

The sample used in this study, denoted as XMBE277 was grown in house using a RIBER V100 molecular beam epitaxy (MBE) system on a (100) semi-insulating InP substrate. Table I shows the epitaxial structures which consist of a $400 \mathrm{~nm}$ highly doped $\mathrm{n}^{+}$$\mathrm{In}_{0.53} \mathrm{Ga}_{0.47} \mathrm{As}$ collector-contact layer on top of an InP substrate. This was followed by a $25 \mathrm{~nm} \mathrm{n}-\mathrm{In}_{0.53} \mathrm{Ga}_{0.47} \mathrm{As}$ collector layer. Then, a $20 \mathrm{~nm}$ undoped $\mathrm{In}_{0.53} \mathrm{Ga}_{0.47} \mathrm{As}$ spacer layer was grown. This spacer layer also prevents the diffusion of dopants into subsequent layers during growth. A $1.3 \mathrm{~nm}$ undoped AlAs barrier, a $4.5 \mathrm{~nm}$ undoped highly-strained $\mathrm{In}_{0.8} \mathrm{Ga}_{0.2} \mathrm{As}$ well, and another $1.3 \mathrm{~nm}$ undoped AlAs barrier formed the double barrier quantum well (DBQW) structure. A further $20 \mathrm{~nm}$ undoped $\mathrm{In}_{0.53} \mathrm{Ga}_{0.47} \mathrm{As}$ spacer layer and a $25 \mathrm{~nm} \mathrm{n}$ $\mathrm{In}_{0.53} \mathrm{Ga}_{0.47} \mathrm{As}$ emitter were grown on top of the DBQW structure. Finally a $45 \mathrm{~nm}$ highly doped $\mathrm{n}^{+}-\mathrm{In}_{0.53} \mathrm{Ga}_{0.47} \mathrm{As}$ emitter-contact layer was grown to facilitate good ohmic-contacts. In order to characterise and optimise the growth conditions especially for the thin highly-strained $\mathrm{In}_{0.8} \mathrm{Ga}_{0.2} \mathrm{As} / \mathrm{AlAs}$ DBQW structure, a double crystal $\mathrm{x}$ ray diffraction (DCXRD) method was used to estimate the various epitaxial layer thicknesses.

TABLE I. The epitaxial structure for sample XMBE277.

\begin{tabular}{|c|c|c|}
\hline Layer & $\begin{array}{l}\text { Thickness } \\
\text { (nm) }\end{array}$ & $\begin{array}{c}\text { Doping } \\
\text { Concentration }(\mathrm{cm} \\
3 \text { ) }\end{array}$ \\
\hline$n^{+}-\mathrm{In}_{053} \mathrm{Ga}_{047} \mathrm{As}$ & 45 & $2.00 \times 10^{19}$ \\
\hline$n$ - $\mathrm{In}_{0.53} \mathrm{Ga}_{0.47} \mathrm{As}$ & 25 & $3.00 \times 10^{18}$ \\
\hline $\mathrm{In}_{0.53} \mathrm{Ga}_{0.47} \mathrm{As}$ & 20 & undoped \\
\hline AlAs & 1.3 & undoped \\
\hline $\mathrm{In}_{0.8} \mathrm{Ga}_{0.2} \mathrm{As}$ & 4.5 & undoped \\
\hline AlAs & 1.3 & undoped \\
\hline $\mathrm{In}_{0.53} \mathrm{Ga}_{0.47} \mathrm{As}$ & 20 & undoped \\
\hline$n-\mathrm{In}_{0.53} \mathrm{Ga}_{0.47} \mathrm{As}$ & 25 & $3.00 \times 10^{18}$ \\
\hline$n^{+}-\mathrm{In}_{0.53} \mathrm{Ga}_{0.47} \mathrm{As}$ & 400 & $1.00 \times 10^{19}$ \\
\hline \multirow{2}{*}{\multicolumn{3}{|c|}{$\begin{array}{l}\text { Semi-insulating } \\
\text { InP }\end{array}$}} \\
\hline & & \\
\hline
\end{tabular}

The RTD emitter lateral dimensions as defined by mask patterns are $1 \mathrm{x} 1 \mu \mathrm{m}^{2}, 1.5 \mathrm{x} 1 \mu \mathrm{m}^{2}, 2 \mathrm{x} 1 \mu \mathrm{m}^{2}, 3 \mathrm{x} 1$ $\mu \mathrm{m}^{2}$ and $4 \mathrm{x} 1 \mu \mathrm{m}^{2}$. However, when subjected to the softreflow technique, the final emitter areas become $1 \mathrm{x} 0.35 \mu \mathrm{m}^{2}, 1.5 \mathrm{x} 0.35 \mu \mathrm{m}^{2}, 2.0 \mathrm{x} 0.35 \mu \mathrm{m}^{2}, 3.0 \mathrm{x} 0.35 \mu \mathrm{m}^{2}$ and $4 \times 0.35 \mu \mathrm{m}^{2}$ respectively. These 5 sub-micrometer dimensions are incorporated in order to determine the dependency of oscillation frequency on mesa area at a later stage of this study. Reducing mesa area is a geometrical way of reducing the RTD capacitance to improve frequency performance. It is also important to observe if there is no degradation in DC performance within the sub-micrometer areas.

It may be worthwhile to mention that the length (1.0 $\mu \mathrm{m}, 1.5 \mu \mathrm{m}, 2.0 \mu \mathrm{m}, 3.0 \mu \mathrm{m}$ and $4.0 \mu \mathrm{m})$ of the emitter is defined by the mask. However, the width of the emitter although initially defined by the mask $(1.0 \mu \mathrm{m})$ during photolithography, its final width is determined after the soft-reflow technique is completed. There was no process induced bias to the fabricated dimensions.

The processing begins with an LOR 3A resist being spin-coated onto the surface of the semiconductor to produce a $300 \mathrm{~nm}$ thick film. This is then followed by the deposition of $200 \mathrm{~nm} \mathrm{Si}_{3} \mathrm{~N}_{4}$ on top of the LOR 3A. LOR 3A lift-off resist is based on polydimethylglutarimide. It is used as an undercut layer underneath the $\mathrm{Si}_{3} \mathrm{~N}_{4}$ and $\mathrm{S} 1805$ photoresist. While having an excellent adhesion to InGaAs, the processing is easy without the need for extra UV exposure. The third layer, Shipley S1805 positive photoresist of about $420 \mathrm{~nm}$ thick is then spin-coated on top of the $\mathrm{Si}_{3} \mathrm{~N}_{4}$. Shipley S1805 belongs to Microposit S1800 series positive photoresist. It is compatible with LOR 3A resist and i-line photolithography. The tri-layer technique is useful for a clean and easy metal lift-off. To define the 1 $\mu \mathrm{m}$ emitter opening, an i-line optical lithography is used to transfer the pattern onto the positive photoresist. The sample is then subjected to the soft-reflow process until the opening shrinks from $1 \mu \mathrm{m}$ down to $350 \mathrm{~nm}$. This sub-micrometer feature is transferred to the $\mathrm{Si}_{3} \mathrm{~N}_{4}$ by $\mathrm{CF}_{4}$ reactive ion-etching, followed by $\mathrm{O}_{2}$ reactive ion-etching (RIE) to strip the LOR 3A resist in order to expose the semiconductor surface. An undercut profile is created by using an MF319 solution to facilitate a clean metal liftoff. Microposit MF-319 is a metal-ion-free developer dedicated for S1800 series positive photoresists, thus the best candidate for S1805 development. Using an immersion technique, excellent resist profile can be obtained.

The sample is then dipped into a diluted Hydrochloric acid solution, $\mathrm{HCl}: \mathrm{H}_{2} \mathrm{O}=1: 1$ for the deoxidation process before metal deposition by thermal evaporation takes place. This is to ensure a good contact between metal and semiconductor surfaces with minimum oxide formation. An emitter metal consisting of Ti/Au $(50 \mathrm{~nm} / 200 \mathrm{~nm})$ is deposited onto the surface of a highly doped $\mathrm{In}_{0.53} \mathrm{Ga}_{0.47} \mathrm{As}$ emitter by thermal evaporation and subsequent lift off in hot N-methyl-2pyrrolidone (NMP) solvent. Fig. 3 describes the first processing step (tri-layer soft-reflow) for the submicrometer RTD.

0018-9383 (c) 2014 IEEE. Personal use of this material is permitted. Permission from IEEE must be obtained for all other users, including reprinting/ republishing this material for advertising or promotional purposes, creating new collective works for resale or redistribution to servers or lists, or reuse of any copyrighted components of this work in other works. DOI:

http://dx.doi.org/10.1109/TED.2014.2322107 


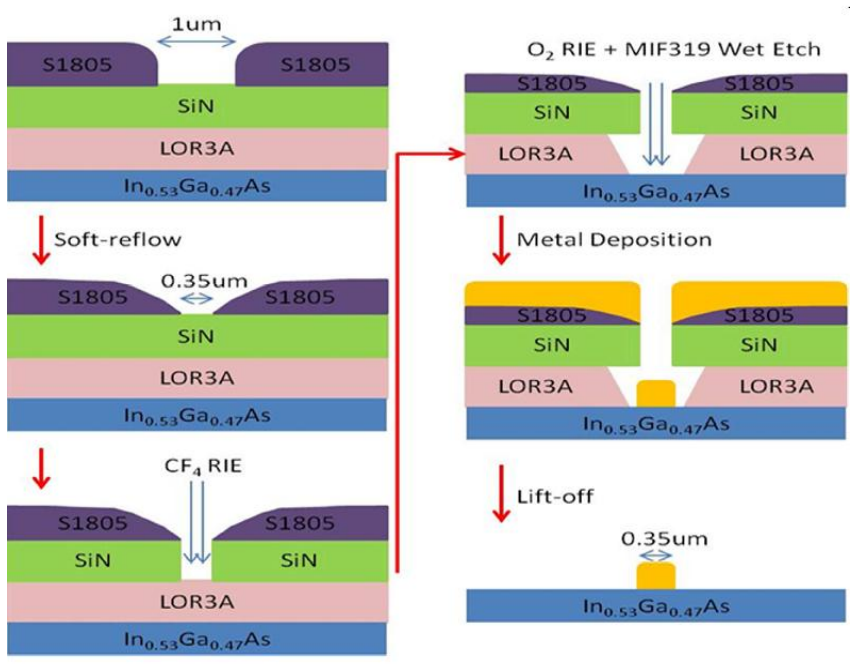

Fig. 3. Illustration of the first processing steps for the submicrometer RTDs.

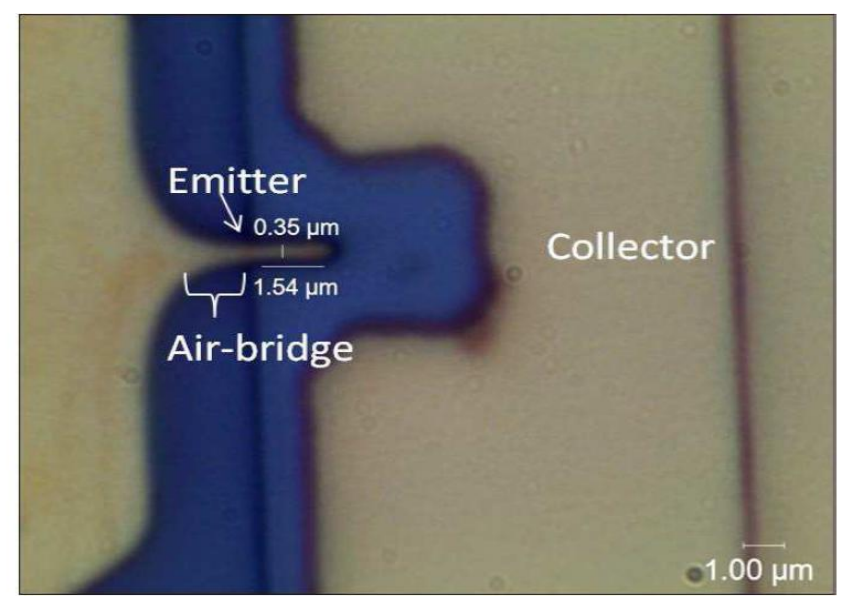

Fig. 4. The completed sub-micrometer RTD with emitter lateral dimension of $\sim 1.54 \mu \mathrm{m}$ (length) and $\sim 0.35 \mu \mathrm{m}$ (width) resulting in a mesa area of $\sim 0.54 \mu \mathrm{m}^{2}$.

Using the emitter metal as an etch mask, the sample is put into $\mathrm{CH}_{4}: \mathrm{H}_{2}$ (1:8) reactive-ion-etching for 20 minutes. The chamber pressure is kept at $40 \mathrm{mTorr}$ at a power of $150 \mathrm{~W}$. This resulted in an anisotropic sidewall $210 \mathrm{~nm}$ down to the collector layer.

Subsequently, wet-etching is performed by applying a positive photoresist and UV-photolithography to define the areas. An Ortho-phosphoric etchant $\left(\mathrm{H}_{2} \mathrm{O}: \mathrm{H}_{3} \mathrm{PO}_{4}: \mathrm{H}_{2} \mathrm{O}_{2}=50: 3: 1\right)$, with an etch rate of $90 \mathrm{~nm}$ per minute is used to etch away about $300 \mathrm{~nm}$ of epilayer materials down to the surface of InP, which acts as an etch-stop layer. The 5 minutes wet-etch will simultaneously provide the necessary undercut through lateral etching for the air-bridge formation. The airbridge structure $(1 \mu \mathrm{m} \times 3 \mu \mathrm{m})$ must be fully opened to avoid high parasitic (parallel) resistance. Both the submicron area and large-area processing used identical process flow during the formation of the air-bridge structures.

Finally, the collector contact is formed by thermal evaporation of $\mathrm{Ti} / \mathrm{Au}(50 \mathrm{~nm} / 500 \mathrm{~nm})$. The bottom (collector) contact is also ohmic, similar to the top emitter contact. A typically completed sub-micrometer RTD is shown in Fig. 4. The schematic cross-section of the RTD is illustrated in Fig. 5, showing two distinct regions of passive and active mesas connected through an air-bridge structure.

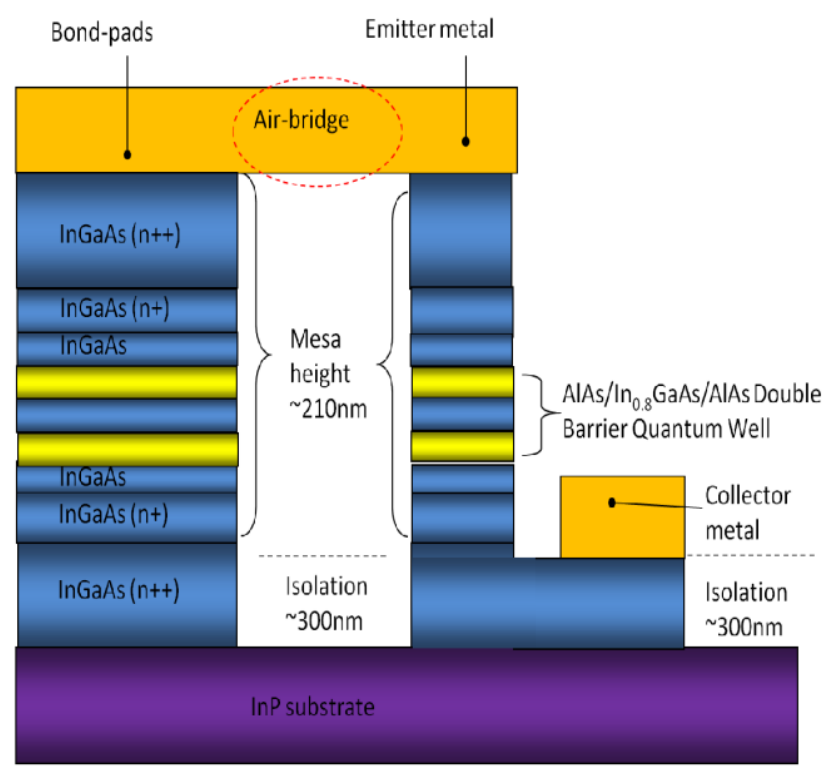

Fig. 5. Schematic illustration of the cross-sectional view of the sub-micrometer RTDs.

\section{RESULTS AND DISCUSSIONS}

Upon completion of the sub-micrometer RTD, room temperature DC characteristics were measured using an HP 4142 DC parameter analyser. The success of the submicrometer processing can be quantitatively determined by device scalability in terms of their peak current densities. For this purpose, large areas RTDs are fabricated together with the sub-micrometer RTDs on the same sample as to minimise process-related errors. Fig. 6 below depicts measured current-voltage characteristics of a large area RTD $\left(28.6 \mu \mathrm{m}^{2}\right)$ and a submicrometer RTD $\left(0.54 \mu \mathrm{m}^{2}\right)$, a nearly $60: 1$ reduction in active area.

0018-9383 (c) 2014 IEEE. Personal use of this material is permitted. Permission from IEEE must be obtained for all other users, including reprinting/ republishing this material for advertising or promotional purposes, creating new collective works for resale or redistribution to servers or lists, or reuse of any copyrighted components of this work in other works. DOI:

http://dx.doi.org/10.1109/TED.2014.2322107 


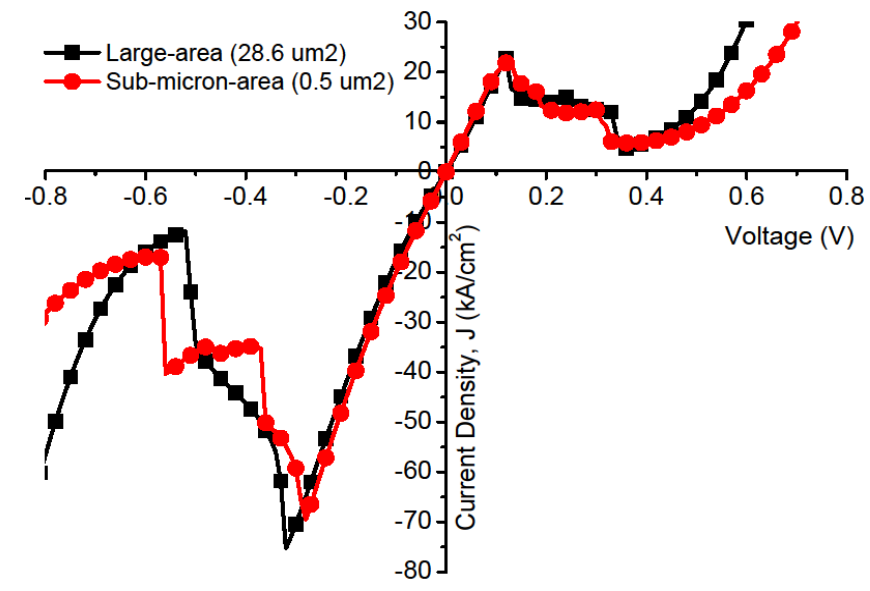

Fig. 6. Measured current-voltage characteristics for sample XMBE277 at room temperature for different mesa sizes. Note the very low value of peak voltage.

In forward bias, the large area RTD has a peak current density of $23 \mathrm{kA} / \mathrm{cm}^{2}$ which occurs at a peak voltage of $120 \mathrm{mV}$. The sub-micrometer RTD has similar peak current density of $22 \mathrm{kA} / \mathrm{cm}^{2}$ at a peak voltage of $130 \mathrm{mV}$. The $10 \mathrm{mV}$ difference in peak voltage is due to the resolution of the step-size in this measurement. In the negative polarity, the large area RTD has a peak current density of $75 \mathrm{kA} / \mathrm{cm}^{2}$ which occurs at a peak voltage of $320 \mathrm{mV}$, and the submicrometer RTD has a peak current density of 69 $\mathrm{kA} / \mathrm{cm}^{2}$ which occurs at peak voltage of $280 \mathrm{mV}$.

Evidently, as the emitter area is scaled down to 0.525 $\mu \mathrm{m}^{2}$ from $28.62 \mu \mathrm{m}^{2}$, the peak voltage $\left(\mathrm{V}_{\mathrm{P}}\right)$ between these two RTDs are almost the same at $\sim 120 \mathrm{mV}$ in forward bias and $\sim 300 \mathrm{mV}$ in reverse direction. This implies that the RTDs are not depleted by the $\mathrm{CH}_{4}$ reactive-ion-etching (RIE) process. Otherwise, the peak voltage for sub-micrometer RTD would have drifted to higher value due to series resistance effects associated with side-wall damage.

Noticeably, the fabricated RTDs have a very low value of peak voltage, $V_{p}$ and in particular in the forward direction, the peak current occurs at a voltage as low as $120 \mathrm{mV}$. Notice that even though too large a peak current, $\mathrm{I}_{\mathrm{P}}$ may be undesirable due to the issue of high power dissipation during circuit implementation, this can be compensated by designing RTD with very low peak voltage, $\mathrm{V}_{\mathrm{P}}$.

The fact that both peak current densities between these two devices are similar, serves as an indicator towards excellent device scalability. A full overview of peak current density as a function of device area is plotted in Fig. 7.

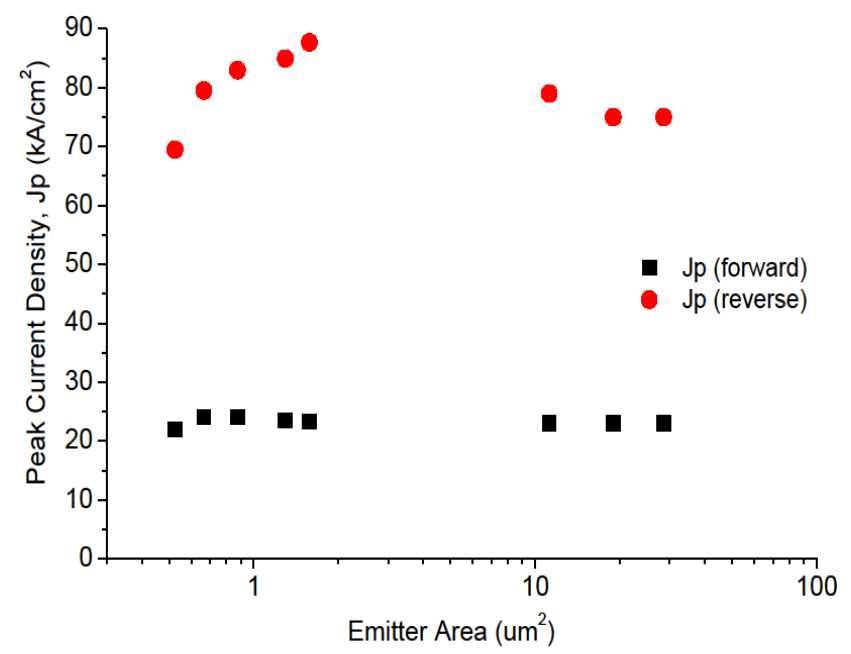

Fig. 7._Measured peak current density against emitter area for sample XMBE277.

Clearly, in the sub-micrometer areas $\left(0.35 \mu \mathrm{m}^{2}\right.$, $0.54 \mu \mathrm{m}^{2}$ and $\left.0.7 \mu \mathrm{m}^{2}\right)$, the RTDs are still able to maintain peak current densities similar to those of the large-areas RTDs (beyond $10 \mu \mathrm{m}^{2}$ ) at roughly 23 $\mathrm{kA} / \mathrm{cm}^{2}$ in the forward direction and around $80 \mathrm{kA} / \mathrm{cm}^{2}$ when negatively biased. This excellent scalability reveals at least two important aspects for this work. Firstly, the new sub-micrometer process is indeed robust, considering there is no degradation in the value of peak current density. This simply means the current adjusts its value corresponding to the changes in the device mesa area.

Secondly the reactive-ion-etching (RIE) process, using $\left(\mathrm{CH}_{4}: \mathrm{H}_{2}\right)$ is working as intended. The importance of using RIE, as opposed to wet-etch at sub-micrometer lateral dimension has been discussed extensively by Tchego et.al [12]. Small area devices usually suffer from high series resistance due to undercut effect known to be caused by lateral etching for emitter size $<10 \mu \mathrm{m}^{2}$. Since the effective emitter area is reduced, this significantly decreases nominal peak current density, $\mathrm{J}_{\mathrm{p}}$.

By introducing a sub-micrometer processing for the RTDs, the $\mathrm{CH}_{4}$ RIE process to obtain an almost vertical or anisotropic side-wall for the device, becomes mandatory. Reactive-ion-etching using ionised gas is beneficial in terms of having an anisotropic side-wall. But this is at the expense of damaging both the side-wall and semiconductor surface [13]. The latter is not a

0018-9383 (c) 2014 IEEE. Personal use of this material is permitted. Permission from IEEE must be obtained for all other users, including reprinting/ republishing this material for advertising or promotional purposes, creating new collective works for resale or redistribution to servers or lists, or reuse of any copyrighted components of this work in other works. DOI:

http://dx.doi.org/10.1109/TED.2014.2322107 
problem in this case since the damaged surface is beneficial to ensure good metal adhesion to the semiconductor during metal contact formation using thermal evaporation.

However, we did find that the side-wall damage degrades the DC performance in terms of lowering the peak-to-valley-current ratio (PVCR) due to increased leakage current through the side-wall [14]. Fig. 8 shows that the experimental PVCR is reduced from 5.0 to 3.8 and 6.3 to 4.1 as the emitter area shrinks from $28.62 \mu \mathrm{m}^{2}$ to $0.54 \mu \mathrm{m}^{2}$ in both forward and reverse direction respectively. Even though the side-wall damage can be controlled and minimised, this has not implemented in this work, at least at this stage. Nevertheless there is also a tendency for the peak to valley current ration to decrease as the lateral sizes decrease, as seen in other work [15] thus the reduction cannot be solely be attributed to the reactive-ion-etching process.

An interesting development in sub-micrometer III-V Esaki Tunnel Diode (ETD) fabrication was reported by Pawlik et.al [16]. Their sub-micrometer radius of $350 \mathrm{~nm}$ is patterned by electron beam lithography but the final radius is reduced to sub-100nm by undercut due to mesa wet-etching. Similar work has been carried out by Romancyzk et.al [17] with modified doping level of ETD layers. Both authors reported no significant degradation in peak-to-valley-current ratio with respect to scaling down of the junction area.

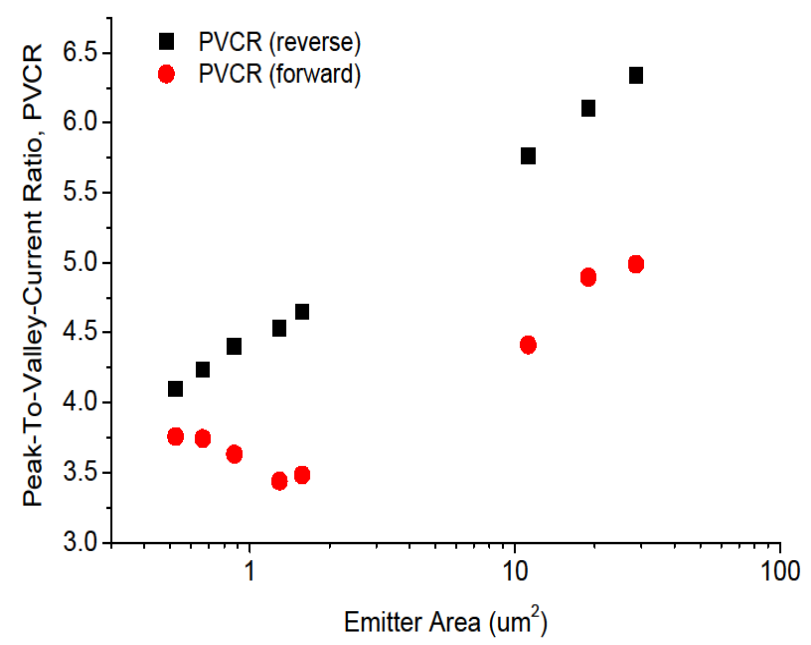

Fig. 8._Measured peak-to-valley-current ratio against emitter area for sample XMBE277.

\section{CONCLUSION}

We have developed an alternative processing technique to obtain sub-micrometer resonant tunnelling diodes without the need for electron beam (EB) lithography or any other complicated techniques. The feature size shrinkage is controllable through parameters such as initial feature size opening, temperature and reflow time.

In our studies, the values of peak current densities, $\mathrm{J}_{\mathrm{P}}$ were uniform throughout the entire range of emitter areas. This technique evidently produced excellent device scalability, good repeatability and a simple process. Most importantly, this new method provides an economical solution to produce resonant tunnelling diodes with sub-micrometer lateral dimensions.

\section{REFERENCES}

[1] Atsushi Teranishi, Kaoru Shizuno, Safumi Suzuki, Masahiro Asada, Hiroki Sugiyama, and Haruki Yokoyama, Fundamental oscillation upto $1.08 \mathrm{THz}$ in resonant tunnelling diodes with high-indium-composition transit layers for reduction of transit delay, IEICE Electronics Express, Vol.9, No.5, 385 (2012).

[2] Michael Feiginov, Cezary Sydlo, Oleg Cojocari, and Peter Meissner, Resonant-tunnelling-diode oscillators operating at frequencies above 1.1 THz, Appl. Phys. Lett. 99, 233506 (2011).

[3] Hidetoshi Kanaya, Hirotaka Shibayama, Riku Sogabe, Safumi Suzuki and Masahiro Asada, Fundamental oscillation up to $1.31 \mathrm{THz}$ in resonant tunnelling diodes with thin well and barriers, Appl. Phys. Express 5, 124101 (2012).

[4] D. Dragoman and M. Dragoman, Terahertz fields and applications, Progress in Quantum Electronics 28, 1 (2004).

[5] Naoyuki Orihashi, Shinnosuke Hattori, Safumi Suzuki, and Masahiro Asada, Experimental and theoretical characteristics of sub-Terahertz and Terehertz oscillations of resonant tunnelling diodes integrated with slot antennas, Japanese Journal of Applied Physics, Vol.44, No.11, 7809 (2005).

[6] M. A. Reed, J. N. Randall, R. J. Aggarwal, R. J. Matyi, T. M. Moore, and A. E. Wetsel, Observation of discrete electronic states in a zero-dimensional semiconductor nanostructure, Phys. Rev. Lett. 60, 535 (1988).

[7] M. T. Bjork, B. J. Ohlsson, C. Thelander, A. I. Persson, K. Deppert, L. R. Wallenberg and L. Samuelson, Nanowire resonant tunneling diodes, Appl. Phys. Lett. 81, 4458 (2002).

[8] S. Tarucha, Y. Hirayama, T. Saku, and T. Kimura, Phys. Rev. B 41, 5459 (1990).

[9] J. Wang, P. H. Beton, N. Mori, H. Buhmann, L. Mansouri, L. Eaves, P. C. Main, T. J. Foster, and M. Henini, Submicrometer resonant tunnelling diodes fabricated by photolithography and selective wet etching, Appl. Phys. Lett. 65, 1124 (1994).

[10] Ka Wa Ian, Michael Exarchos and Mohamed Missous, Fabrication of 250-nm T-gate In52Al48As-In70Ga30As pHEMT using a novel solvent reflow technique at room temperature, in 9th International Conference on Advanced Semiconductor Devices \& Microsystems, ASDAM 2012,

0018-9383 (c) 2014 IEEE. Personal use of this material is permitted. Permission from IEEE must be obtained for all other users, including reprinting/ republishing this material for advertising or promotional purposes, creating new collective works for resale or redistribution to servers or lists, or reuse of any copyrighted components of this work in other works. DOI:

http://dx.doi.org/10.1109/TED.2014.2322107 
Smolenice, Slovakia, 11-15 Nov. 2012 (IEEE Electron

Devices Society, 2012), pp. 99-102.

[11] Ka Wa Ian, Michael Exarchos and Mohamed Missous, A novel low temperature soft reflow process for the fabrication of deep-submicron $(<0.35 \mu \mathrm{m})$ T-gate pseudomorphic high electron mobility transistor structures, Nanotechnology 24, 055202 (2013).

[12] A. Tchego. B. Muenstermann, C. Gutsche, A. Poloczek, K. Blekker, W. Prost, F. J. Tegude, Scalable high current density RTDs with low series resistance, in 22nd International Conference on Indium Phosphide \& Related Materials, IPRM 2010, Kagawa, Japan, 31 May - 4 June 2010, (Institute of Electrical and Electronics Engineers, 2010), pp. 1-4.

[13] S. Murad, M. Rahman, N. Johnson, S. Thoms, S. P. Beaumont, and C. D. W. Wilkinson, Dry etching damage in III-V semiconductors, J. Vac. Sci. Technol. B, Vol. 14, No. 6, pp. 3658 (1996).

[14] Gary Ternent and Douglas J. Paul, SPICE modeling of the scaling of resonant tunneling diodes and the effects of sidewall leakage, IEEE Transactions on Electron Devices, Vol.59, No.12, 3555 (2012).

[15] Bo Su, V. J. Goldman, M. Santos, and M. Shayegan, Resonant tunneling in submicron doublebarrier heterostructures, Appl. Phys. Lett. 58, 747 (1991).

[16] D. Pawlik, B. Romancyzk, P. Thomas, S. Rommel, M. Edirisooriya, R. Contreras-Guerrero, R. Droopad, W. Y. Loh, M. H. Wong, K. Majumdar, W. E. Wang, P. D. Kirsch, and R. Jammy, Benchmarking and improving III-V Esaki diode performance with a record $2.2 \mathrm{MA} / \mathrm{cm}^{2}$ peak current density to enhance TFET drive current, in IEEE International Electron Devices Meeting, IEDM 2012, San Francisco, USA, 10-13 Dec. 2012, (Institute of Electrical and Electronics Engineers, 2012), pp. 27.1.1 - 27.1.3.

[17] B. Romanczyk, P. Thomas, D. Pawlik, S. L. Rommel1, W.-Y. Loh, M. H. Wong, K. Majumdar, W.-E. Wang and P. D. Kirsch, Benchmarking current density in staggered gap In0.53Ga0.47As/GaAs0.5Sb0.5 heterojunction Esaki tunnel diodes, Appl. Phys. Lett. 102, 213504 (2013).

0018-9383 (c) 2014 IEEE. Personal use of this material is permitted. Permission from IEEE must be obtained for all other users, including reprinting/ republishing this material for advertising or promotional purposes, creating new collective works for resale or redistribution to servers or lists, or reuse of any copyrighted components of this work in other works. DOI:

http://dx.doi.org/10.1109/TED.2014.2322107 